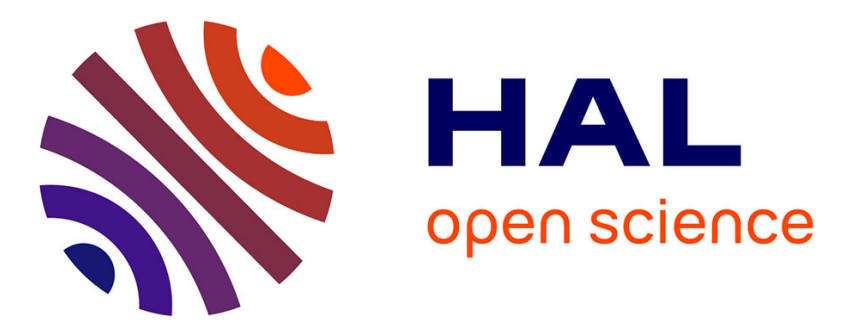

\title{
Les pratiques et les espaces funéraires dans l'Antiquité: état de la recherche, réalités du corpus examiné et orientations du dossier
}

Frédérique Blaizot

\section{- To cite this version:}

Frédérique Blaizot. Les pratiques et les espaces funéraires dans l'Antiquité : état de la recherche, réalités du corpus examiné et orientations du dossier. Gallia - Archéologie de la France antique, 2009, Pratiques et espaces funéraires de la Gaule durant l'Antiquité, 66 (1), pp.1-14. 10.3406/galia.2009.3371. hal-01910998

\section{HAL Id: hal-01910998 \\ https://hal.science/hal-01910998}

Submitted on 7 Jan 2020

HAL is a multi-disciplinary open access archive for the deposit and dissemination of scientific research documents, whether they are published or not. The documents may come from teaching and research institutions in France or abroad, or from public or private research centers.
L'archive ouverte pluridisciplinaire HAL, est destinée au dépôt et à la diffusion de documents scientifiques de niveau recherche, publiés ou non, émanant des établissements d'enseignement et de recherche français ou étrangers, des laboratoires publics ou privés.

\section{(ㅇ)(1) $\$$}

Distributed under a Creative Commons Attribution - NonCommercial - NoDerivatives 44.0 


\title{
LES PRATIQUES ET LES ESPACES FUNÉRAIRES DANS L'ANTIQUITÉ
}

\author{
État de la recherche, réalités du corpus examiné \\ et orientations du dossier
}

\author{
Frédérique BLAIZOT
}

\begin{abstract}
C'est avec un grand enthousiasme et sans doute un brin d'inconscience que nous avons accepté, en février 2004, la proposition de Paul Van Ossel de consacrer un dossier de la revue Gallia aux pratiques funéraires en Gaule romaine ${ }^{1}$. Un projet d'une telle ampleur était très ambitieux ; nous avons ainsi courageusement entrepris de dépouiller Gallia Informations et les Bulletins scientifiques régionaux (BSR) des Services régionaux de l'archéologie, et commencé à contacter des collègues de chaque région, avant de nous rendre compte que l'entreprise était considérable, voire vouée à l'échec. Il nous est apparu en effet très rapidement que l'état d'avancement de la recherche variait énormément d'une région à l'autre et que, dans la grande majorité des cas, le travail réalisé sur les données disponibles au coup par coup n'était pas toujours suffisamment abouti pour être repris sans un énorme travail de fond afin d'être intégré dans le dossier tel que nous l'avions conçu. La partie sud-est
\end{abstract}

1. Les auteurs remercient P. Van Ossel, directeur de Gallia jusqu'en 2007, pour la confiance qu'il nous a accordée, et l'Inrap pour son soutien.

Dans cette étude, nous avons largement exploité les données inédites recueillies par l'ensemble de nos collègues au cours de fouilles préventives (Inrap, Archéodunum, Service archéologique municipal de Lyon) ; nous tenons à saluer le travail formidable qu'ils réalisent sur le terrain, ainsi que celui des services régionaux de l'archéologie auxquels revient la lourde responsabilité des décisions.

Nous sommes également reconnaissantes envers Anne Pariente, directrice du Service archéologique municipal de Lyon, d'avoir mis à notre disposition la documentation élaborée par son service (plan masse des opérations archéologiques reportées sur les plans cadastraux), et envers Jean-Michel Degueule, photographe au musée de la Civilisation gallo-romaine de Lyon, qui a réalisé les clichés des céramiques. de la France, qui a bénéficié très tôt de l'investissement d'un certain nombre de personnes sur le sujet, se trouvait au final la mieux à même de fournir le plus rapidement les éléments en adéquation avec l'esprit dans lequel nous souhaitions traiter le sujet. Précisons que le besoin de définir une archéologie funéraire de l'Antiquité s'est manifesté dès le milieu des années 1980 dans la région (Nécropoles à incinération du Haut-Empire, 1987) et que ces dernières années, Valérie Bel, Laurence Tranoy, Christine Bonnet, Alain Wittmann et moi-même formons une équipe qui s'est fortement impliquée sur des sites funéraires dont le champ chronologique couvre La Tène finale, le Haut-Empire et l'Antiquité tardive. Notre collaboration nous a entraînés à aborder la question des pratiques et des espaces funéraires dans des perspectives plus larges que nous n'aurions été amenés à le faire individuellement, stimulés aussi en cela par nos formations différentes et par les contextes géographiques variés sur lesquels nous sommes intervenus.

Le dossier actuel porte donc principalement sur le centre et le sud-est de la Gaule (fig. 1), notamment les actuelles régions Rhône-Alpes (les Alpes et la vallée du Rhône), Languedoc-Roussillon, Provence, la région de Toulouse, le Massif central représenté par l'Auvergne et l'Aveyron et enfin, plus ponctuellement, sur la partie orientale de la région Centre ${ }^{2}$. L'aire chronologique est centrée sur

2. Ce dossier s'appuie en très grande partie sur des données inédites issues des rapports de fouilles conservés dans les Services régionaux de l'archéologie. Pour des raisons de place, ces rapports ne sont pas cités en bibliographie. 


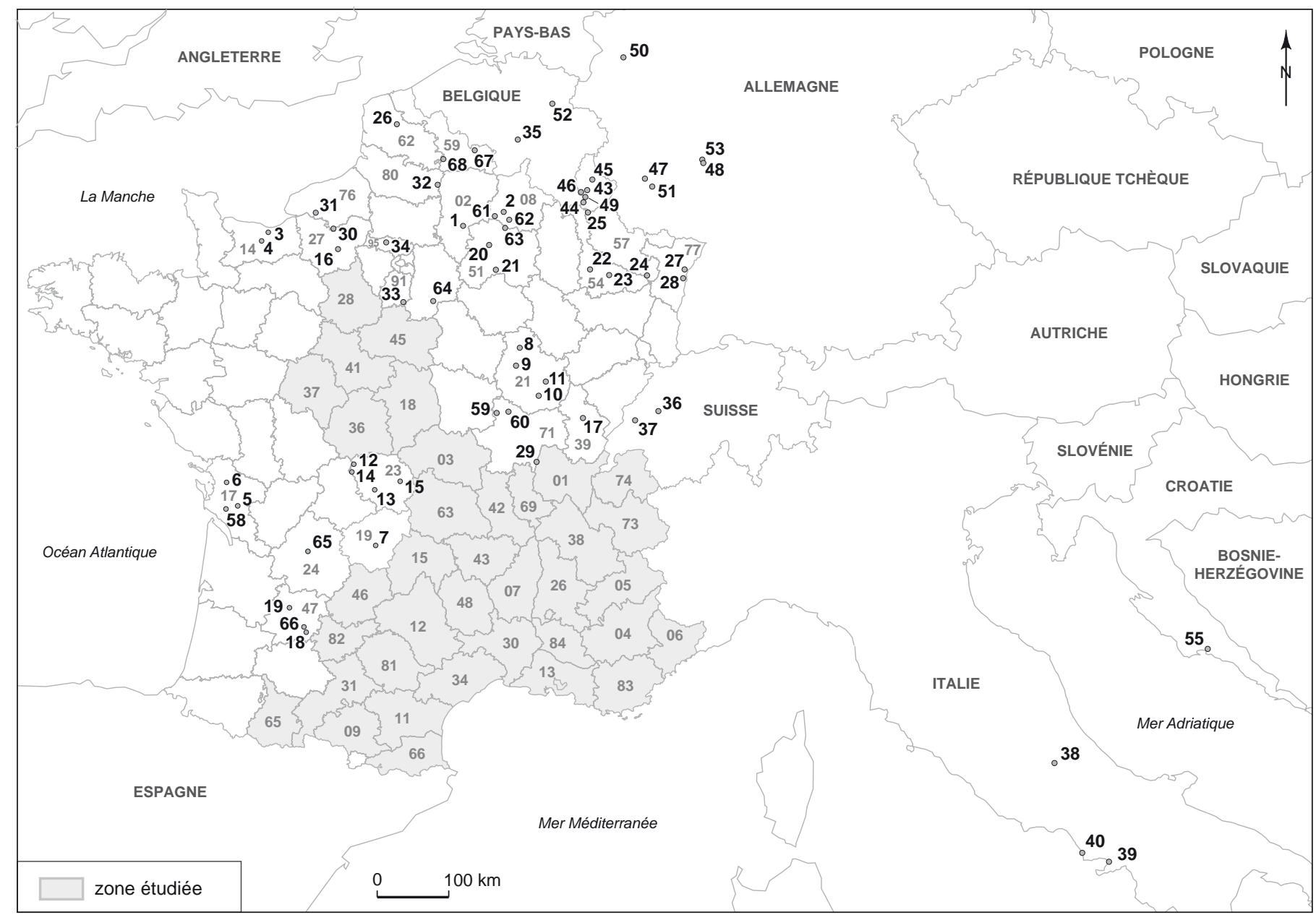

Fig. 1 - Secteurs géographiques étudiés (en grisé) et sites mentionnés dans le texte situés hors de la zone d'étude (DAO : G. Macabéo, Inrap).

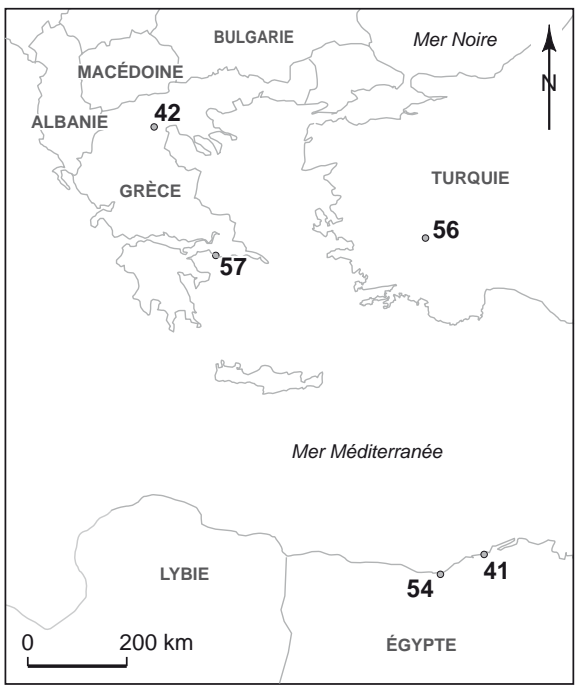




\begin{tabular}{|c|c|c|c|}
\hline \multicolumn{3}{|c|}{ Sites étudiés } & \multirow{2}{*}{$\begin{array}{c}\text { Site hors } \\
\text { zone d'étude }\end{array}$} \\
\hline $\begin{array}{c}\text { Département ou } \\
\text { pays }\end{array}$ & Commune & Lieu-dit & \\
\hline Aisne (02) & Limé & Les Sables & 1 \\
\hline Ardennes (08) & Acy-Romance & & 2 \\
\hline Ardennes (08) & Ménil-Annelles & & 61 \\
\hline Ardennes (08) & Ville-sur-Retourne & & 62 \\
\hline Calvados (14) & Hérouvillette & & 3 \\
\hline Calvados (14) & Saint-Martin-de-Fontenay & & 4 \\
\hline Charente-Maritime (17) & Saintes & & 5 \\
\hline Charente-Maritime (17) & Muron & Champs-Rougis & 6 \\
\hline Charente-Maritime (17) & Saint-Romain-de-Benet & Pirelongue & 58 \\
\hline Corrèze (19) & Saint-Martial-de-Gimel & Chaux de Coudert & 7 \\
\hline Côte-d'Or (21) & Nod-sur-Seine & & 8 \\
\hline Côte-d'Or (21) & Alise-Sainte-Reine & & 9 \\
\hline Côte-d'Or (21) & Meuilley & Gratte-Dos & 10 \\
\hline Côte-d'Or (21) & Dijon & Rue Turgot & 11 \\
\hline Creuse (23) & Vareilles & Fontvieille & 12 \\
\hline Creuse (23) & Pontarion & Les Sagnes & 13 \\
\hline Creuse (23) & Saint-Maurice-la-Souterraine & La Betoulle & 14 \\
\hline Creuse (23) & Saint-Priest & & 15 \\
\hline Dordogne (24) & Périgueux & & 65 \\
\hline Essonne (91) & Buno-Bonnevaux & Chantambre & 33 \\
\hline \begin{tabular}{|l|} 
Eure (27) \\
\end{tabular} & Évreux & & 16 \\
\hline Jura (39) & Poligny & Au Velours & 17 \\
\hline Lot-et-Garonne (47) & Boé & Bordeneuve-de-Bory & 18 \\
\hline Lot-et-Garonne (47) & Mas-d'Agenais (Le) & & 19 \\
\hline Lot-et-Garonne (47) & Agen & & 66 \\
\hline Marne (51) & Avenay-Val-d'Or & La Planche & 20 \\
\hline Marne (51) & Vassimont-et-Chapelaine & Ouche Jaco & 21 \\
\hline Marne (51) & Bétheniville & & 63 \\
\hline \begin{tabular}{|l|} 
Meurthe-et-Moselle (54) \\
\end{tabular} & Villey-Saint-Étienne & & 22 \\
\hline Meurthe-et-Moselle (54) & Varangéville & & 23 \\
\hline Moselle (57) & Saint-Quirin & & 24 \\
\hline \begin{tabular}{|l|} 
Moselle (57) \\
\end{tabular} & Audun-le-Tiche & & 25 \\
\hline Nord (59) & Bavay & Près Aulnoys, La Fache & 67 \\
\hline Nord (59) & Raillencourt-Sainte-Olle & & 68 \\
\hline Pas-de-Calais (62) & Thérouanne & Les Oblets & 26 \\
\hline Bas-Rhin (67) & Reichstett & & 27 \\
\hline Bas-Rhin (67) & Strasbourg & Sainte-Barbe & 28 \\
\hline Sâone-et-Loire (71) & Mâcon & Les Cordiers & 29 \\
\hline Sâone-et-Loire (71) & Saint-Léger-sous-Beuvray & Bibracte & 59 \\
\hline Sâone-et-Loire (71) & Autun & & 60 \\
\hline Seine-Maritime (76) & Caudebec-lès-Elbeuf & & 30 \\
\hline Seine-Maritime (76) & Vatteville-la-Rue & & 31 \\
\hline Seine-et-Marne (77) & Montereau-Fault-Yonne & La Terre aux Moines & 64 \\
\hline Somme (80) & Monchy-Lagache & & 32 \\
\hline Val-d'Oise (95) & Épiais-Rhus & & 34 \\
\hline Allemagne & Wederath-Belginum & & 47 \\
\hline Allemagne & Mayence & Weisenau & 48 \\
\hline Allemagne & Krefeld-Gellep & & 50 \\
\hline Allemagne & Siesbach & & 51 \\
\hline Allemagne & Mainz & Eichelstein & 53 \\
\hline \begin{tabular}{|l} 
Belgique \\
\end{tabular} & Arsimont & & 35 \\
\hline Belgique & Berlingen & & 52 \\
\hline Croatie & Split & Salona & 55 \\
\hline Égypte & Alexandrie & & 41 \\
\hline Égypte & El Alamein & & 54 \\
\hline Grèce & Athènes & & 57 \\
\hline Italie & Aquilée & & 38 \\
\hline Italie & Pompéi & & 39 \\
\hline Italie & Ostie & & 40 \\
\hline \begin{tabular}{|l} 
Luxembourg \\
\end{tabular} & Septfontaines-Deck & & 43 \\
\hline \begin{tabular}{|l|} 
Luxembourg \\
\end{tabular} & Lamadelaine & & 44 \\
\hline Luxembourg & Feulen & & 45 \\
\hline Luxembourg & Bonnert & & 46 \\
\hline \begin{tabular}{|l|} 
Luxembourg \\
\end{tabular} & Clémency & & 49 \\
\hline Macédoine & Vergina & & 42 \\
\hline Suisse & Avenches & En Chaplix & 36 \\
\hline Suisse & Avenches & Porte de l'Ouest-Longeaigue & 36 \\
\hline Suisse & Avenches & Avenches-Port & 36 \\
\hline Suisse & Yverdon-les-Bains & Le Pré de la Cure & 37 \\
\hline Turquie & Hiérapolis & & 56 \\
\hline
\end{tabular}


le Haut-Empire, mais le second âge du Fer et l'Antiquité tardive ont aussi été abordés afin d'éclairer certains aspects portant sur les questions de continuité et de discontinuité des pratiques funéraires.

Nous regrettons au terme de ce travail que nos collègues provençaux, attelés à d'autres tâches et plus particulièrement aux nécessaires publications de monographies importantes sur le sujet (Aix-en-Provence et Fréjus notamment), n'aient pas pu se consacrer à ce projet ; nous tenons en tout cas à remercier chaleureusement Nuría Nin et Claude Raynaud qui ont accepté de mettre très tôt à notre disposition leurs manuscrits sur Aix-en-Provence (Nin et al., 2006) et LunelViel (Raynaud dir., sous presse), afin que nous puissions recueillir des données pour notre enquête.

Il n'est pas question ici de retracer exhaustivement l'histoire de la recherche sur les «sépultures et nécropoles » de l'Antiquité depuis les origines, d'en souligner les errements et d'en pointer les impasses. Le bilan général qui s'en dégage est que les sources funéraires ont surtout été utilisées par les chercheurs pour répondre à des questions qui ne relèvent pas de leur champ spécifique. Cette orientation n'est toutefois pas propre à l'archéologie de l'Antiquité : les expressions funéraires de toutes périodes ont abondamment servi de vivier pour alimenter l'étude des aspects divers et variés des sociétés du passé. Pour ce qui concerne l'époque qui nous occupe, les données issues du domaine funéraire s'inscrivirent très tôt dans une perspective évolutionniste et diffusionniste, dans l'objectif d'apporter des éclairages sur les modalités de deux événements historiques majeurs qui ont marqué la Gaule : la romanisation, puis la christianisation (Déchelette, 1927 ; Nock, 1932 ; Cumont, 1949 ; Turcan, 1958 ; Audin, 1960 ; Van Doorselaer, 1967 ; Toynbee, 1971 ; Hatt, 1951 et 1986). Dans ce cadre, les données des structures et des espaces funéraires, abordées dans leurs grandes lignes et par certains aspects, furent supposées documenter les changements sociaux distillés par les textes, et s'épanouirent dans les domaines artistiques, religieux, philosophiques et économiques (Nock, 1932 ; Cumont, 1942 ; Hatt, 1951 et 1986 ; Audin, 1960 ; Visscher, 1963 ; Hoptkins, 1983 ; Prieur, 1986 ; Hinard dir., 1987 ; Haffner, 1989 ; Galliou, 1989). Les rites funéraires, par ailleurs souvent minutieusement décryptés (Van Doorselaer, 1967), sont perçus comme des invariants fondamentaux d'une population et, bien sûr, d'un ensemble de croyances.

À partir du milieu des années 1980 apparaissent de nouvelles perspectives pour l'archéologie funéraire, avec notamment le développement des études des assemblages et des dispositifs sépulcraux (Duday, 1981 ; Pétrequin, 1984 ;
Duday, Masset dir., 1987), et celui de l'analyse des os brûlés (Duday, 1987 ; Grévin, 1990). Celles-ci, conjuguées à l'explosion de l'archéologie préventive, contribuent à recentrer la réflexion sur ce qui constitue le véritable sujet des pratiques funéraires, à savoir le traitement du défunt et de ses restes. Pour les régions qui nous occupent, d'importants ensembles funéraires sont exhumés (fig. 2 et 3 ) : pour la moitié sud de la France à Lyon (Rhône, notamment la Favorite), SaintPaul-Trois-Châteaux (Drôme), Fréjus (Var), Martigues (Bouches-du-Rhône), Aix-en-Provence (Bouches-duRhône) et la Brillanne (Alpes-de-Haute-Provence), pour les régions plus centrales à Roanne (Loire), ClermontFerrand (Puy-de-Dôme, avec le Pâtural), Argentomagus/ Saint-Marcel (Indre), Chalais (Indre), Lazenay (Cher), Pontarion (Creuse) et Faverdines (Cher), mais aussi hors de notre zone d'étude, le site de Chantambre à BunoBonnevaux (Essonne), celui de la Croix-Guillaume à SaintQuirin (Moselle), Avenches en Suisse, etc. Dès lors, on assiste à un renouvellement complet du sujet. Trois thèses majeures sont engagées, l'une récemment publiée sur un ensemble funéraire de la vallée du Rhône (Bel et al., 2002), la deuxième sur le site de la Favorite et la topographie funéraire de la capitale des Gaules (Tranoy, 1995b), puis la dernière sur le site de Chantambre dans l'Essonne (Murail, 1996), dont les apports furent décisifs dans l'essor de la problématique actuelle. Parallèlement, les études de cas ne cessent de se multiplier, donnant lieu à des réflexions collectives (Nécropoles à incinération du Haut-Empire, 1987). Parmi elles, le colloque de 1991, Incinérations et inhumations dans l'Occident romain aux trois premiers siècles de notre ère, illustre la première tentative de mise en évidence de la variabilité des pratiques à l'échelle de la Gaule et des territoires périphériques, même si dans ses visées, la réflexion porte encore sur la question de l'identité romaine des pratiques funéraires appréhendées de manière partielle. En 1992, c'est sous l'angle de l'organisation territoriale et donc socio-économique, que la variabilité du domaine funéraire gallo-romain est abordée dans le cadre d'un autre colloque, dirigé par A. Ferdière (Ferdière dir., 1993), tandis que l'année suivante, un colloque tenu à Mayence tente de faire le point sur l'ensemble des aspects du domaine funéraire révélés par des données archéologiques récentes dans (presque) toutes les provinces (Struck dir., 1993). À une échelle plus générale, on constate que l'accent porte alors plutôt sur les rapports sociaux que sur les changements (von Hesberg, Zanker dir., 1987 ; Morris, 1992 ; Davies, 1999 ; Pearce et al. dir., 2000) et sur le rôle social des pratiques funéraires (Schmitt-Pantel, 1982 ; Lavagne, 1987 ; Landes dir., 
2002) déjà abordé par P.-A. Février pour l'Antiquité tardive (Février, 1977), tandis que les chercheurs spécialistes des textes s'interrogent sur le sens religieux des rituels funéraires et les représentations de la mort chez les Romains (Février, 1983 et 1984 ; Maurin, 1984 ; Scheid, 1984 et 2005). Les années 1990 et le début des années 2000 s'enrichissent d'études de cas et de bilans d'étapes ; outre les titres déjà cités, notons entre autres, la publication des Sagnes à Pontarion dans la Creuse (Lintz dir., 2001), de Fléré-laRivière à Esvres en Indre-et-Loire (Ferdière, Villard, 1993), de la voie de l'Océan à Lyon (Frascone, 1999), de SainteBarbe à Marseille (Moliner et al., 2003), de Vernègues dans les Bouches-du-Rhône (Chapon et al., 2004), des cimetières de nourrissons de Sallèles-d'Aude dans l'Aude (Duday et al., 1995) et de Champ Madame à Beaumont dans le Puy-deDôme (Alfonso, Blaizot dir., 2004), de Soumaltre à Aspiran dans l'Hérault (Thernot et al., 2004), de plusieurs sites champenois (Stead et al., 2006), d'autres de la région Centre (Ensembles funéraires gallo-romains de la région Centre, 2006) et des ensembles funéraires méridionaux d'Aix-en-Provence (Nin et al., 2006). En dehors de la France, émergent celles du site de Wederath/Belginum en Allemagne (Haffner, 1989), de Septfontaines-Dëckt (Polfer, 1996) et de l'oppidum du Titelberg de Lamadelaine au Luxembourg (Metzler-Zens et al. dir., 1999), d'En Chaplix à Avenches (Castella et al., 1999 et 2002). Plusieurs colloques récents sont à souligner, comme celui de Durham dont l'objectif était de mettre en évidence la diversité des pratiques funéraires dans leurs différents aspects pour l'ensemble des provinces romaines (Pearce et al. dir., 2000) ou celui sur la Gaule Belgique (Geoffroy, Barbé dir., 2001), sans en oublier deux autres, plurichronologiques, qui incluent des articles portant sur les pratiques funéraires de l'Antiquité (Baray dir., 2004 ; Baray et al. dir., 2007). Prochainement, les publications des sites de Lunel-Viel (Raynaud dir., sous presse), de Nîmes (Bel et al., 2008) et de Fréjus (par C. Gébara et I. Béraud), celles de deux projets collectifs de recherche en cours dont l'un porte sur les ensembles funéraires ruraux du bassin de Clermont-Ferrand (Blaizot dir.) et l'autre sur ceux de Champagne-Ardenne (Paresys dir.) ainsi, espérons-le, qu'au moins les travaux menés sur les ensembles périurbains de Saintes (B. Farrago et J.-P. Baigl) et sur le site de la CroixGuillaume à Saint-Quirin en Moselle, contribueront à offrir de nouveaux corpus de données primaires.

Même si de nombreuses monographies restent en souffrance et que les études de cas sont toujours insuffisamment publiées, il paraît utile de faire le point sur la question, objectif vers lequel tend ce dossier sous l'éclairage d'un secteur géographique. Cette définition du dossier est plus réaliste et surtout plus conforme à l'état actuel de la recherche que le serait une synthèse des pratiques funéraires de l'ensemble du monde gallo-romain. Ces dix dernières années en effet, la mise en commun de nos résultats sur de nombreuses séries a permis de réexaminer une fois de plus les faits sous un angle différent de celui qui, jusqu'alors, conditionnait le raisonnement. S'est notamment imposée l'évidence selon laquelle les manifestations funéraires et l'organisation des morts de l'Antiquité étaient probablement plus complexes qu'elles n'apparaissaient de prime abord, et que l'ensemble des paramètres, à commencer par la définition fonctionnelle des structures dans le cadre de la cérémonie funéraire, méritait d'être reconsidéré (Tranoy, 2000 ; Bel, 2004 ; Blaizot, Tranoy, 2004 ; Richier, 2005 ; Blaizot, Bonnet, 2007 ; Blaizot et al., 2007 ; Bel et al., 2008). Cette perspective constitue d'ailleurs le fil majeur de ce dossier.

Cette rapide rétrospective montre que les orientations historiques, économiques et philosophiques à large portée ont, pour un temps au moins, cédé la place à des questionnements qui procèdent plus directement des données archéologiques. De même, l'assimilation du monde des morts au monde des vivants, ou plus exactement l'idée que l'organisation des uns traduit l'organisation sociale des autres, semblent aujourd'hui devoir être nuancées, dès lors que l'on examine les faits sur la durée et en relation avec les données historiques ou sociales issues d'autres sources. Le système funéraire paraît constitué d'autant de variables, indépendantes les unes des autres et de celles qui se rapportent à d'autres aspects d'une société, même si toutes se croisent et s'articulent. Encore faut-il être en mesure de saisir chacun des systèmes dans sa spécificité. À cet égard, sont actuellement privilégiées, dans les recherches sur le domaine funéraire, les questions relatives aux modalités de genèse, d'organisation et d'abandon des espaces funéraires, aux gestes et aux pratiques comme expressions du système funéraire et à la mise en évidence de la variabilité de l'ensemble de ces manifestations.

C'est dans cet esprit que nous avons choisi de privilégier deux approches fondamentales. La première traite des pratiques funéraires dans une conception dynamique (les pratiques dans l'unité des funérailles) et la seconde de la relation que la société entretient avec l'espace des morts (l'organisation des ensembles funéraires au sein d'un territoire). Nous proposons enfin, à partir de cette documentation, plusieurs pistes de réflexion : variabilité intra- et interrégionale des pratiques, mise en évidence des étapes 


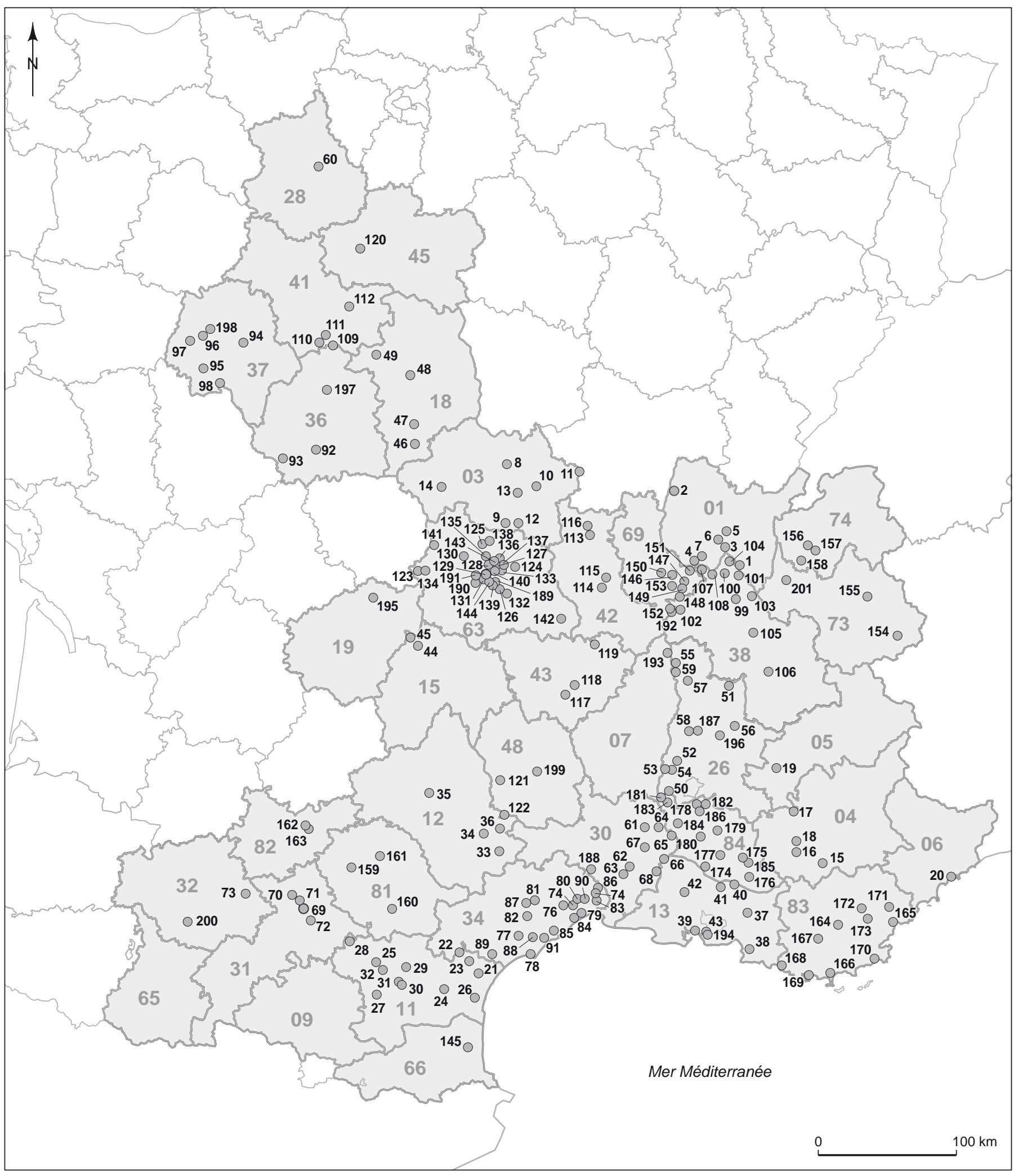

Fig. 2 - Sites du centre et du sud-est de la Gaule mentionnés dans le texte (DAO : G. Macabéo, Inrap). 


\begin{tabular}{|c|c|c|c|c|c|c|c|}
\hline Département & Commune & Lieu-dit & \begin{tabular}{|c} 
zone \\
étudiée
\end{tabular} & Département & Commune & Lieu-dit & $\begin{array}{c}\text { zone } \\
\text { étudiée }\end{array}$ \\
\hline Ain (01) & Briord & Les Plantées & 1 & Drôme (26) & Allan & Grangeneuve & 54 \\
\hline Ain (01) & Replonges & La Madeleine & 2 & Drôme (26) & Ponsas & Fontager & 55 \\
\hline Ain (01) & Ambutrix & & 3 & Drôme (26) & Die & & 56 \\
\hline Ain (01) & Boisse (La) & Villa des Vernes & 4 & Drôme (26) & Clérieux & Les Sablières & 57 \\
\hline Ain (01) & Ambronay & Sur la Côte & 5 & Drôme (26) & Chabrillan & Saint-Martin & 58 \\
\hline Ain (01) & Château-Gaillard & & 6 & Drôme (26) & Érôme & & 59 \\
\hline Ain (01) & Béligneux & & 7 & Drôme (26) & Crest & Bourbousson & 187 \\
\hline Allier (03) & Chemilly & & 8 & \multirow{2}{*}{ Drôme (26) } & \multirow{2}{*}{ Saillans } & \multirow{2}{*}{$\begin{array}{l}\text { Monument d'Aulus } \\
\text { Pompeius Fronto }\end{array}$} & \multirow{2}{*}{196} \\
\hline Allier (03) & Cognat-Lyonne & & 9 & & & & \\
\hline Allier (03) & Chavroches & & 10 & Eure-et-Loir (28) & Chartres & Pôle Synéo & 30 \\
\hline Allier (03) & Chassenard & & 11 & Gard (30) & Tresques & Courac & 61 \\
\hline Allier (03) & Vichy & & 12 & Gard (30) & Nîmes & 59, avenue Jean-Jaurès & 62 \\
\hline Allier (03) & Varennes-sur-Allier & & 13 & Gard (30) & Nîmes & 78, avenue Jean-Jaurès & 62 \\
\hline Allier (03) & Saint-Angel & & 14 & Gard (30) & Nîmes & $\begin{array}{l}\text { 594, avenue du Maréchal- } \\
\text { Juin }\end{array}$ & 62 \\
\hline Alpes-de-Haute-Provence (04) & Riez & & 15 & & Nîmes & Carmes & 62 \\
\hline Alpes-de-Haute-Provence (04) & Brillanne (La) & Saint-Martin & 16 & Gard (30) & Nîmes & Îlot Grill & 62 \\
\hline Alpes-de-Haute-Provence (04) & Bevons & Castels-Bévon & 17 & Gard (30) & Nîmes & Magaille est & 62 \\
\hline Alpes-de-Haute-Provence (04) & Lurs & & $\frac{18}{19}$ & Gard (30) & Nîmes & Mas Carbonnel & 62 \\
\hline \begin{tabular}{|l|} 
Hautes-Alpes (05) \\
Alpes-Maritimes (06)
\end{tabular} & \begin{tabular}{|l} 
Bâtie-Montsaléon (La) \\
Nice
\end{tabular} & & $\frac{19}{20}$ & Gard (30) & Nîmes & Mas des Abeilles II.3 & 62 \\
\hline \begin{tabular}{|l} 
Alpes-Maritimes (06) \\
Ardèche (07)
\end{tabular} & $\begin{array}{l}\text { Nice } \\
\text { Andance }\end{array}$ & Piol de Cimiez & $\frac{20}{193}$ & Gard (30) & Nîmes & Mas des Abeilles II.6 & 62 \\
\hline \begin{tabular}{|l} 
Aude (11) \\
\end{tabular} & Narbonne & Rue Jules-Verne & $\frac{193}{21}$ & Gard (30) & Nîmes & Mas des Abeilles VII & 62 \\
\hline Aude (11) & Narbonne & Bonne Source & 21 & Gard (30) & Nîmes & Mas de Vignoles IV & 62 \\
\hline Aude (11) & Narbonne & Razimbaud-Rue d'Artois & 21 & Gard (30) & Nîmes & Mas de Vignoles IX & 62 \\
\hline Aude (11) & Narbonne & Hotel-Dieu & 21 & Gard (30) & Nîmes & Montaury, Nîmes & 62 \\
\hline Aude (11) & Mailhac & Caylar & 22 & Gard (30) & Nîmes & Place des Arènes & 62 \\
\hline Aude (11) & Sallèles-d'Aude & & 23 & Gard (30) & Nîmes & 85 , route d'Alès & 62 \\
\hline \multirow{2}{*}{ Aude (11) } & \multirow{2}{*}{$\begin{array}{l}\text { Saint-Laurent-de-la- } \\
\text { Cabrerisse }\end{array}$} & & \multirow[b]{2}{*}{24} & Gard (30) & Nîmes & 53 , route de Beaucaire & 62 \\
\hline & & & & Gard (30) & Nîmes & 113 , route de Beaucaire, & 62 \\
\hline Aude (11) & Bram & Le Village & 25 & Gard (30) & Nîmes & 21, rue Séguier & 62 \\
\hline Aude (11) & Sigean & Pech Maho & 26 & Gard (30) & Nîmes & Rue Henri-Révoil & 62 \\
\hline Aude (11) & Villelongue-d'Aude & & 27 & Gard (30) & Nîmes & Saint-André-de-Codols & 62 \\
\hline Aude (11) & Montmaur & Puech Calvel & 28 & Gard (30) & Milhaud & La Condamine & 63 \\
\hline Aude (11) & Carcassonne & Monquier & 29 & Gard (30) & Milhaud & $\begin{array}{l}\text { Careiron et Pesquier } \\
\text { (corpus VB) }\end{array}$ & 63 \\
\hline Aude (11) & Pomas & Lagaste & 30 & Gard (30) & Laudun & La Brèche & 64 \\
\hline Aude (11) & Rouffiac-d'Aude & Lagaste & $\begin{array}{l}31 \\
32\end{array}$ & Gard (30) & Roquemaure & La Ramière & 65 \\
\hline \begin{tabular}{|l|} 
Aude (11) \\
Avevron (12)
\end{tabular} & \begin{tabular}{|l} 
Montréal \\
Hosnitalet-du-L arzac (L')
\end{tabular} & L'Espitalet & $\frac{32}{33}$ & Gard (30) & Aramon & Le Paradis & 66 \\
\hline Aveyron (12) & Millau & La Graufesenque & 34 & \begin{tabular}{|l|} 
Gard (30) \\
\end{tabular} & Vers-Pont-du-Gard & L'Estel & 67 \\
\hline \begin{tabular}{|l|} 
Aveyron (12) \\
\end{tabular} & Rodez & Notre-Dame-du-Bon-Accueil & 35 & Gard (30) & Beaucaire & & 68 \\
\hline Aveyron (12) & Rodez & Jardin du Séminaire & 35 & Gard (30) & Gailhan & Oppidum & 188 \\
\hline \multirow{2}{*}{ Aveyron (12) } & \multirow{2}{*}{$\begin{array}{l}\text { Roque-Sainte-Marguerite } \\
\text { (La) }\end{array}$} & & \multirow[b]{2}{*}{36} & Haute-Garonne (31) & Toulouse & Rue Achille-Viadieu & 69 \\
\hline & & & & Haute-Garonne (31) & Toulouse & Impasse Augustin-Thierry & 69 \\
\hline Bouches-du-Rhône (13) & Aix-en-Provence & ZAC Sextius Mirabeau & 37 & Haute-Garonne (31) & Toulouse & Métro François-Verdier & 69 \\
\hline Bouches-du-Rhône (13) & Marseille & Sainte-Barbe & 38 & Haute-Garonne (31) & Toulouse & Caserne Niel & 69 \\
\hline Bouches-du-Rhône (13) & Marseille & Bassin de Carénage & 38 & Haute-Garonne (31) & Beauzelle & Le Barricou & 70 \\
\hline Bouches-du-Rhône (13) & Port-de-Bouc & & 39 & Haute-Garonne (31) & Blagnac & Grand Noble 3 & 71 \\
\hline Bouches-du-Rhône (13) & Roque-d'Anthéron (La) & & 40 & Haute-Garonne (31) & Castanet-Tolosan & & 72 \\
\hline \multirow{2}{*}{ Bouches-du-Rhône (13) } & \multirow{2}{*}{ Vernègues } & Les Communaux de & 41 & Gers (32) & Touget & & 73 \\
\hline & & Saint-Cézaire & 41 & Gers (32) & Mirande & & 200 \\
\hline Bouches-du-Rhône (13) & Vernègues & L'Héritière & 41 & Hérault (34) & Lunel-Viel & Le Verdier & 74 \\
\hline Bouches-du-Rhône (13) & Mouriès & Le Cagalou & 42 & Hérault (34) & Montpellier & Gallière & 75 \\
\hline Bouches-du-Rhône (13) & Martigues & La Gatasse & 43 & Hérault (34) & Montpellier & Malbosc & 75 \\
\hline Bouches-du-Rhône (13) & Martigues & «Temple» du Ponteau, & 194 & Hérault (34) & Montpellier & Les Cauquillous & 75 \\
\hline Doderies-ud-krionte (10) & iviartigues & Seneymes & 194 & Hérault (34) & Murviel-lès-Montpellier & Sur les Jardins & 76 \\
\hline Cantal (15) & Chavagnac & & 44 & Hérault (34) & Murviel-lès-Montpellier & Les Combes & 76 \\
\hline Cantal (15) & Champagnac & & 45 & Hérault (34) & Valros & La Vigne de Bioaux & 77 \\
\hline Cher (18) & Faverdines & Les Vernes & 46 & Hérault (34) & Agde & Le Peyrou & 78 \\
\hline Cher (18) & Bruère-Allichamps & Les Varnes & 47 & Hérault (34) & Agde & Le Bousquet & 78 \\
\hline Cher (18) & Bourges & Lazenay & 48 & Hérault (34) & Lattes & La Céreirède & 79 \\
\hline Cher (18) & Bourges & Saint-Martin-des-Champs & 48 & Hérault (34) & Castelnau-le-Lez & Navitau & 80 \\
\hline Cher (18) & Vierzon & Vieux Domaine & 49 & Hérault (34) & Saint-André-de-Sangonis & Lagarel & 81 \\
\hline Corrèze (19) & Saint-Merd-les-Oussines & Les Cars & 195 & Hérault (34) & Aspiran & Soumaltre & 82 \\
\hline Drôme (26) & Saint-Paul-Trois-Châteaux & Le Valladas & 50 & Hérault (34) & Lansargues & Les Quatre-Carrières & 83 \\
\hline Drôme (26) & Saint-Laurent-en-Royans & & 51 & Hérault (34) & Villeneuve-lès-Maguelone & Domenove 3 & 84 \\
\hline Drôme (26) & Montboucher-sur-Jabron & Le Patis & 52 & Hérault (34) & Balaruc-les-Bains & Chemin Haut & 85 \\
\hline Drôme (26) & Châteauneuf-du-Rhône & La Labre & 53 & Hérault (34) & Villetelle & Ambrussum & 86 \\
\hline
\end{tabular}




\begin{tabular}{|c|c|c|c|}
\hline Département & Commune & Lieu-dit & $\begin{array}{c}\text { zone } \\
\text { étudiée }\end{array}$ \\
\hline Hérault (34) & Clermont-l'Hérault & Peyre Plantade & 87 \\
\hline Hérault (34) & Pinet & Roc Haute & 88 \\
\hline Hérault (34) & Nissan-lez-Enserune & Enserune & 89 \\
\hline Hérault (34) & Crès (Le) & Maumarin & 90 \\
\hline Hérault (34) & Mèze & Saint-Martin & 91 \\
\hline Indre (36) & Saint-Marcel & Champ de I'Image & 92 \\
\hline Indre (36) & Chalais & & 93 \\
\hline Indre (36) & Levroux & & 197 \\
\hline Indre-et-Loire (37) & Esvres & Vaugrignon & 94 \\
\hline Indre-et-Loire (37) & Esvres & Fléré-la-Rivière & 94 \\
\hline Indre-et-Loire (37) & Tavant & 42, rue Grande & 95 \\
\hline Indre-et-Loire (37) & Langeais & Les Béziaux & 96 \\
\hline Indre-et-Loire (37) & Saint-Patrice & La Pérrée & 97 \\
\hline Indre-et-Loire (37) & Celle-Saint-Avant (La) & & 98 \\
\hline Indre-et-Loire (37) & Cinq-Mars-la-Pile & & 198 \\
\hline Isère (38) & Saint-Jean-de-Soudain & & 99 \\
\hline Isère (38) & Optevoz & & 100 \\
\hline Isère (38) & Creys-Mépieu & & 101 \\
\hline Isère (38) & Vienne & Rue Vimaine & 102 \\
\hline Isère (38) & Vienne & Charavel & 102 \\
\hline Isère (38) & Aoste & La Planche & 103 \\
\hline Isère (38) & Montalieu-Vercieu & Chalépont & 104 \\
\hline Isère (38) & Voiron & La Brunerie & 105 \\
\hline Isère (38) & Varces-Allières-et-Ricet & Lachar & 106 \\
\hline Isère (38) & Varces-Allières-et-Ricet & Drabuyard & 106 \\
\hline Isère (38) & Villette-d'Anthon & Les Sablons & 107 \\
\hline Isère (38) & Villette-d'Anthon & $\begin{array}{l}\text { Contournement de } \\
\text { Janneyrias/Villette }\end{array}$ & 107 \\
\hline Isère (38) & Saint-Romain-de-Jalionas & Verna & 108 \\
\hline Loir-et-Cher (41) & Gièvres & L'Érable & 109 \\
\hline Loir-et-Cher (41) & Billy & Les Cloubeaux & 110 \\
\hline Loir-et-Cher (41) & Gy-en-Sologne & Les Mahis & 111 \\
\hline Loir-et-Cher (41) & Gy-en-Sologne & Le Grand Saulé, & 111 \\
\hline Loir-et-Cher (41) & Neung-sur-Beuvron & Le Chemin du Plessis & 112 \\
\hline Loire (42) & Roanne & & 113 \\
\hline Loire (42) & Saint-Laurent-la-Conche & Bel Air & 114 \\
\hline Loire (42) & Feurs & Rue Saint-Antoine & 115 \\
\hline Loire (42) & Mably & Bonvert & 116 \\
\hline Haute-Loire (43) & \begin{tabular}{|l|} 
Saint-Christophe-sur- \\
Dolaison
\end{tabular} & & 117 \\
\hline Haute-Loire (43) & Brives-Charensac & & 118 \\
\hline Haute-Loire (43) & Bas-en-Basset & & 119 \\
\hline Loiret (45) & Orléans & \begin{tabular}{|l|} 
rue du Faubourg \\
Bourgogne
\end{tabular} & 120 \\
\hline Loiret (45) & Orléans & Centre de Conférence & 120 \\
\hline Lozère (48) & Banassac & Champ del as & 121 \\
\hline Lozère (48) & Rozier (Le) & & 122 \\
\hline Lozère (48) & Lanuéjols & Pré-des-Clastres & 199 \\
\hline Puy-de-Dôme (63) & Giat & & 123 \\
\hline Puy-de-Dôme (63) & Lezoux & Les Religieuses & 124 \\
\hline Puy-de-Dôme (63) & Combronde & & 125 \\
\hline Puy-de-Dôme (63) & Martres-de-Veyre (Les) & & 126 \\
\hline Puy-de-Dôme (63) & Malintrat & Chaniat & 127 \\
\hline Puy-de-Dôme (63) & Gerzat & Rochefort & 128 \\
\hline Puy-de-Dôme (63) & Gerzat & Le Pâtural & 128 \\
\hline Puy-de-Dôme (63) & Clermont-Ferrand & Jardin botanique & 129 \\
\hline Puy-de-Dôme (63) & Clermont-Ferrand & Brézet & 129 \\
\hline Puy-de-Dôme (63) & Clermont-Ferrand & Aulnat-Gandaillat & 129 \\
\hline Puy-de-Dôme (63) & Clermont-Ferrand & La Grande Borne & 129 \\
\hline Puy-de-Dôme (63) & Clermont-Ferrand & Rue de l'Oradou & 129 \\
\hline Puy-de-Dôme (63) & Clermont-Ferrand & Rue des Paulines & 129 \\
\hline Puy-de-Dôme (63) & Clermont-Ferrand & Bds Auradou/Lafayette & 129 \\
\hline Puy-de-Dôme (63) & Clermont-Ferrand & La Pardieu & 129 \\
\hline Puy-de-Dôme (63) & Clermont-Ferrand & Petites Gravanches & 129 \\
\hline Puy-de-Dôme (63) & Pulvérières & Cime des Bruyères & 130 \\
\hline Puy-de-Dôme (63) & Beaumont & Champ-Madame & 131 \\
\hline Puy-de-Dôme (63) & Pardines & La Maison Blanche & 132 \\
\hline
\end{tabular}

\begin{tabular}{|c|c|c|c|}
\hline Département & Commune & Lieu-dit & $\begin{array}{c}\text { zone } \\
\text { étudiée }\end{array}$ \\
\hline Puy-de-Dôme (63) & Pont-du-Château & Champ Bussière & 133 \\
\hline Puy-de-Dôme (63) & Pont-du-Château & Champ Lamet & 133 \\
\hline Puy-de-Dôme (63) & Cendre (Le) & Gondole & 189 \\
\hline Puy-de-Dôme (63) & Voingt & & 134 \\
\hline Puy-de-Dôme (63) & Riom & Bromon-Lamothe & 135 \\
\hline Puy-de-Dôme (63) & Chappes & La Buyre & 136 \\
\hline Puy-de-Dôme (63) & Martres-d'Artière (Les) & Les Quaires & 137 \\
\hline Puy-de-Dôme (63) & Martres-d'Artière (Les) & Champ Chalatras & 138 \\
\hline Puy-de-Dôme (63) & Saint-Myon & & 139 \\
\hline Puy-de-Dôme (63) & Aulnat & Aulnat-Gandaillat & 140 \\
\hline Puy-de-Dôme (63) & Charensat & & 141 \\
\hline Puy-de-Dôme (63) & Saint-Just & & 142 \\
\hline Puy-de-Dôme (63) & Saint-Beauzire & Biopôle & 143 \\
\hline Puy-de-Dôme (63) & Pérignat-lès-Sarliève & & 144 \\
\hline Puy-de-Dôme (63) & $\begin{array}{l}\text { Saint-Genès- } \\
\text { Champanelle }\end{array}$ & Col de Ceyssat & 190 \\
\hline Puy-de-Dôme (63) & Orcines & $\begin{array}{l}\text { Sanctuaire du } \\
\text { Puy-de-Dôme }\end{array}$ & 191 \\
\hline Pyrénées-Orientales (66) & Perpignan & Ruscino & 145 \\
\hline Rhône (69) & Lyon & & 146 \\
\hline Rhône (69) & Saint-Priest & Les Feuillis & 147 \\
\hline Rhône (69) & Saint-Priest & Les Luèpes & 147 \\
\hline Rhône (69) & Marennes & Saint-Fréjus & 148 \\
\hline Rhône (69) & Marennes & Le Pillon & 148 \\
\hline Rhône (69) & Corbas & Boulevard Urbain-Sud & 149 \\
\hline Rhône (69) & Tassin-Ia-Demi-Lune & La Raude & 150 \\
\hline Rhône (69) & Meyzieu & Le Trillet & 151 \\
\hline Rhône (69) & Saint-Romain-en-Gal & & 152 \\
\hline Rhône (69) & Sainte-Colombe & Place de l'Égalité & 192 \\
\hline Rhône (69) & Saint-Fons & Balan & 153 \\
\hline Savoie (73) & Lanslevillard & & 154 \\
\hline Savoie (73) & Aime & Le Replat & 155 \\
\hline Savoie (73) & Aix-les-Bains & & 201 \\
\hline Haute-Savoie (74) & Annecy & ZAC Albert & 156 \\
\hline Haute-Savoie (74) & Veyrier-du-Lac & & 157 \\
\hline Haute-Savoie (74) & Viuz-la-Chiésaz & & 158 \\
\hline Tarn (81) & Montans & & 159 \\
\hline Tarn (81) & Castres & & 160 \\
\hline Tarn (81) & Albi & Mas d'Agenais & 161 \\
\hline Tarn-et-Garonne (82) & Cayrac & Les Plaines & 162 \\
\hline Tarn-et Garonne (82) & Réalville & Larsou & 163 \\
\hline $\operatorname{Var}(83)$ & Cabasse & La Guérine & 164 \\
\hline $\operatorname{Var}(83)$ & Cabasse & La Cabasse & 164 \\
\hline $\operatorname{Var}(83)$ & Fréjus & Le Pauvadou & 165 \\
\hline Var (83) & Fréjus & Saint-Lambert & 165 \\
\hline $\operatorname{Var}(83)$ & Hyères & Olbia-de-Provence & 166 \\
\hline $\operatorname{Var}(83)$ & Garéoult & Rue Louis-Cauvin & 167 \\
\hline $\operatorname{Var}(83)$ & Saint-Cyr-sur-Mer & & 168 \\
\hline \begin{tabular}{|l|}
$\operatorname{Var}(83)$ \\
\end{tabular} & Toulon & & 169 \\
\hline $\operatorname{Var}(83)$ & Croix-Valmer (La) & & 170 \\
\hline Var (83) & Bagnols-en-Forêt & & 171 \\
\hline $\operatorname{Var}(83)$ & Draguignan & & 172 \\
\hline Var (83) & Arcs (Les) & & 173 \\
\hline Vaucluse (84) & Cavaillon & & 174 \\
\hline Vaucluse (84) & Apt & & 175 \\
\hline Vaucluse (84) & Sannes & & 176 \\
\hline Vaucluse (84) & Gordes & & 177 \\
\hline Vaucluse (84) & Roaix & & 178 \\
\hline Vaucluse (84) & Mormoiron & & 179 \\
\hline Vaucluse (84) & Carpentras & & 180 \\
\hline Vaucluse (84) & Lapalud & Les Girardes & 181 \\
\hline Vaucluse (84) & Vaison-la-Romaine & Pommerol & 182 \\
\hline Vaucluse (84) & Bollène & Pont de Pierre & 183 \\
\hline Vaucluse (84) & Orange & Fouches Vieilles & 184 \\
\hline Vaucluse (84) & Saignon & & 185 \\
\hline Vaucluse (84) & Séguret & & 186 \\
\hline
\end{tabular}


des rites funéraires, fonction du mobilier, continuité et discontinuité des pratiques funéraires de La Tène finale au Bas-Empire et rôle social des pratiques funéraires.

Il n'échappera à personne que nous avons délibérément tourné le dos au plan «classique » qui aurait consisté à présenter les faits en juxtaposant les composantes funéraires (le traitement du corps, les structures, le mobilier par catégorie, etc). Celui-ci nous est en effet apparu inadapté à rendre compte des rites en tant qu'ensemble structuré, de l'appareil funéraire en tant que dispositif, sans occulter la complexité des gestes funéraires sur la durée. On cherchera donc en vain des bilans successifs sur l'instrumentum, les dépôts alimentaires, etc. Nous avons plutôt décidé de refermer la boîte de Pandore pour sélectionner les thèmes en fonction de leur caractère novateur et, comme nous l'avons précisé ci-dessus, surtout pour tenter d'appréhender la dynamique du système funéraire antique.

A contrario, certains pourront être surpris par le caractère très analytique de ce dossier, que nous avons voulu largement argumenté et illustré. Cette abondance de démonstrations et d'études de cas s'explique par le fait que les données sur lesquelles s'appuie la réflexion sont, en majeure partie, inédites et également par le fait que cette réflexion repose sur une manière nouvelle d'analyser les vestiges archéologiques du monde funéraire. Notons par exemple que le premier souci que l'on rencontre est d'identifier correctement les structures et, avant tout, de les décrire et de les aborder de manière raisonnée. C'est pourquoi nous nous sommes attachés à présenter le plus précisément possible les situations archéologiques, qui interviennent alors comme argumentation plutôt que comme illustration.

Nous avons dépouillé un très grand nombre de travaux réalisés dans la région d'étude et il nous faut tout d'abord souligner l'énorme potentiel que représentent les fouilles préventives, très peu exploitées tant dans nos régions que dans celles que nous avons survolées lors de notre enquête initiale à l'échelle de la France. On constate, en revanche, que la recherche sur les pratiques funéraires gallo-romaines est actuellement quasi absente de l'archéologie programmée et qu'elle est peu prisée dans les universités qui proposent un enseignement en archéo-anthropologie. Trois thèses proviennent en effet d'universités qui n'ont pas cette spécialité à leur programme : deux de l'université Paris-I, dont l'une fut présentée en 2005 par Raphaël Durand (2005) et dont l'autre est en cours par Nathalie Baills-Talbi (Sentiment de l'enfance et reconnaissance sociale : la place des jeunes enfants, 0-4 ans, dans la société gallo-romaine, I ${ }^{e r}$ s. av. notre ère-IV $V^{e}$. $a p r$. notre ère). La troisième a été soutenue en 2003 à l'uni- versité de Franche-Comté à Besançon par Lydie Joan sur les ensembles funéraires régionaux (L’Archéologie funéraire en Franche-Comté antique du $I^{e r}$ siècle av. J.-C. au V apr. J.-C). Mise à part celle, en cours, d'Anne Richier menée à l'université de Marseille (Les Vivants face à leurs morts : évolution des gestes et des comportements funéraires durant l'Antiquité - $I^{e r} s$. av. au VII ${ }^{e}$ s. apr. J.-C. - à partir de sites provençaux, de l'archéoanthropologie à l'histoire sociale), le sujet est boudé depuis une quinzaine d'années par le milieu anthropologique.

Pour ce qui concerne l'archéologie préventive, nous retenons de l'ensemble des rapports de fouilles examinés deux constantes qui nuisent à l'obtention de données. La première rend compte d'une difficulté à construire une problématique funéraire propre à l'Antiquité. En effet, si les structures sont généralement correctement abordées du point de vue méthodologique et technique, les questionnements sont morcelés, procédant plutôt des diverses disciplines impliquées par les artefacts en présence, chacune développant ses objectifs indépendamment des autres, mais rarement selon une problématique commune. Globalement, la problématique reste plus céramologique ou « ostéocentrique » que funéraire. Force est de constater en effet que les progrès réalisés dans la prise en compte des os brûlés ont eu parfois l'effet pervers de faire oublier l'objectif principal de la fouille, qui est l'étude du dispositif et du fonctionnement des structures dans leur contexte. La deuxième constante, en partie liée à la première, est que les études engagées sur un ensemble funéraire sont rarement le résultat d'un choix délibéré, mais dépendent des spécialistes disponibles et des moyens alloués à l'opération. Les lacunes sont particulièrement criantes pour ce qui concerne le «petit mobilier », la faune, les restes végétaux et alimentaires, quelle que soit leur importance pour l'étude du site, disciplines pour lesquelles l'archéologie préventive manque de personnel. Enfin, dans les espaces funéraires fouillés sur une surface importante, par exemple en contexte périurbain, il est très rare que les études aient été conduites sur l'ensemble, faute de moyens, l'analyse des structures liées à la crémation en étant particulièrement "dévoreuse ». Ainsi, il existe un très grand nombre de structures et de sites partiellement étudiés, voire mis en caisse après le traitement primaire des vestiges (lavage, tri et stockage), quand ce n'est pas directement après la fouille (contenu des sacs de prélèvement non tamisés). À Lyon, c'est le cas de quelques sites, dont certains d'envergure (celui de Villa Montel à Vaise par exemple) et de nombreuses structures exhumées dans le cadre de diagnostics. 


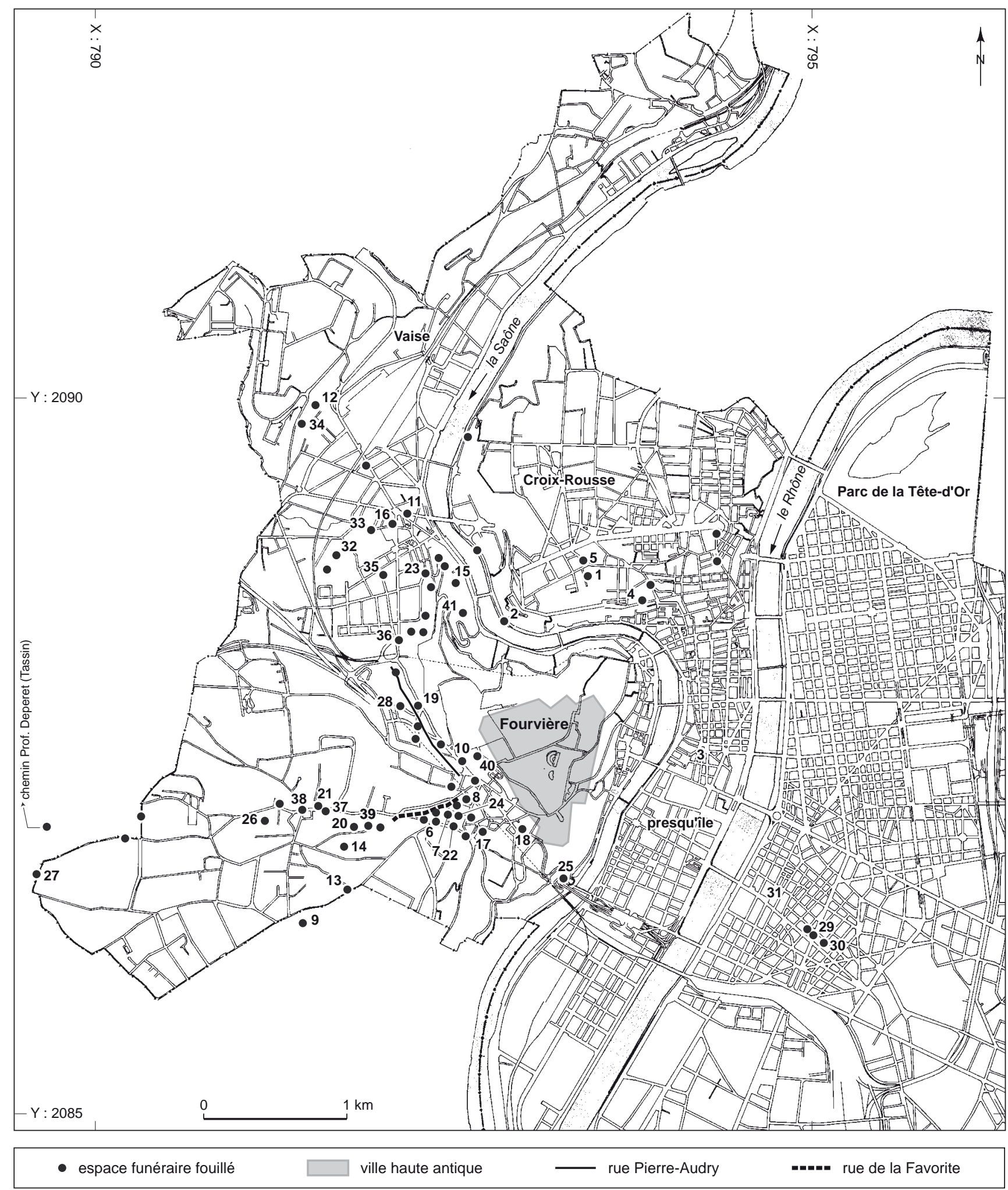




\begin{tabular}{|c|c|c|}
\hline Arrondissement & Voie ou site & $\mathbf{N}^{\circ}$ \\
\hline $1^{\mathrm{er}}$ & Montée de la Butte Quai Saint-Vincent & 1 \\
\hline $1^{\mathrm{er}}$ & 7-9-11, rue des Chartreux & 2 \\
\hline $2^{e}$ & Place des Célestins & 3 \\
\hline $4^{e}$ & Clinique Saint-Charles & 4 \\
\hline $4^{e}$ & Rue des Chartreux & 5 \\
\hline $5^{e}$ & La Favorite I et II & 6 \\
\hline $5^{e}$ & 20 , rue de la Favorite & 7 \\
\hline $5^{\mathrm{e}}$ & Mausolées de Trion & 8 \\
\hline $5^{e}$ & Les Bruyères et Clos Nouvelet & 9 \\
\hline $5^{e}$ & 117-121, rue Pierre-Audry & 10 \\
\hline $9^{e}$ & 4-6, rue du Mont-d'Or & 11 \\
\hline $9^{e}$ & Voie de l'Océan-Boulevard du périphérique Nord & 12 \\
\hline $5^{\mathrm{e}}$ & 62, rue du Commandant-Charcot (les Roses/le Sextant) & 13 \\
\hline $5^{e}$ & 19, rue des Granges & 14 \\
\hline $9 \mathrm{e}$ & 29-30, quai Arloing & 15 \\
\hline $9 \mathrm{~g}$ & Villa Montel, $41-43$, rue du Bourbonnais & 16 \\
\hline $5^{e}$ & Saint-Irénée & 17 \\
\hline $5^{e}$ & Saint-Just & 18 \\
\hline $5^{e}$ & 77-79, rue Pierre-Audry (Contrescarpe) & 19 \\
\hline $5^{e}$ & 53-54, avenue du Point-du-Jour & 20 \\
\hline $5^{e}$ & 62, avenue du Point-du-Jour & 21 \\
\hline $5^{e}$ & 50, avenue du Point-du-Jour & 37 \\
\hline $5^{e}$ & 64-66, avenue du Point-du-Jour & 38 \\
\hline $5^{e}$ & 51, avenue du Point-du-Jour & 39 \\
\hline $5^{e}$ & Rue Trouvée & 22 \\
\hline $9^{e}$ & 47, rue du Chapeau-Rouge & 23 \\
\hline $5^{e}$ & 19-21, rue des Fossés-de-Trion & 24 \\
\hline $5^{e}$ & Saint-Laurent-de-Choulans & 25 \\
\hline $5^{e}$ & 41 , rue Joliot-Curie & 26 \\
\hline $5^{e}$ & 207, rue Joliot-Curie (Clos des Cèdres) & 27 \\
\hline $5^{e}$ & 54, rue Pierre-Audry & 28 \\
\hline $5^{e}$ & Place du Cardinal-Gerlier & 40 \\
\hline $7^{e}$ & Rues Rochette-Chevrier & 29 \\
\hline $7^{e}$ & Rue de la Madeleine & 30 \\
\hline $7^{e}$ & Mausolée des Acceptii, rue de Marseille & 31 \\
\hline $9^{e}$ & 9 , rue du Docteur-Horand & 32 \\
\hline $9^{e}$ & Îlot Mas & 33 \\
\hline $9 e$ & 30-32, rue de Bourgogne & 34 \\
\hline $9^{e}$ & 38, rue du Sergent-Berthet & 35 \\
\hline $9^{e}$ & 51-53, rue du Sergent-Berthet & 36 \\
\hline $9^{e}$ & Pont du Général-Kœnig & 41 \\
\hline
\end{tabular}

$\longleftarrow$

Fig. 3 - Les ensembles funéraires du Haut-Empire à Lyon. Les points sur la carte désignent tous les sites; seuls ceux mentionnés dans le texte sont numérotés. Les sites numérotés sans points sont ceux dont la localisation n'est pas précisée car découverts aux XVIII et XIX ${ }^{e}$ s. (L. Tranoy, DAO : G. Macabéo, Inrap; fond de plan : Service archéologique municipal de Lyon).
L'une des difficultés que nous avons rencontrées dans les régions sélectionnées pour ce dossier se rapporte aux disparités de la documentation de référence. Celles-ci touchent la taille des corpus, la répartition chronologique des sites funéraires, la part de l'inhumation et de la crémation durant le Haut-Empire ainsi que les perspectives dans lesquelles les travaux furent menés. Ces contrastes régionaux nuisent à la mise en évidence des spécificités et de la variabilité régionales et interrégionales des pratiques funéraires. Par ailleurs, les sites ayant été en grande majorité exhumés dans le cadre préventif (donc de manière opportuniste tant sur les contextes que sur les surfaces d'investigation), il n'est même pas certain que les trois premières disparités relevées traduisent, pour les sociétés du passé, des pratiques ou des organisations régionales différentes.

La situation actuelle de l'archéologie funéraire antique dans le secteur examiné est donc très contrastée. Sans trop trahir, je l'espère, les bilans détaillés fournis par mes collègues (V. Bel, C. Bonnet, P. Georges, L. Tranoy, A. Wittmann), il semble que l'on peut souligner les points suivants.

C'est dans les régions Rhône-Alpes et Provence-AlpesCôte d'Azur qu'ont été fouillés des ensembles funéraires périurbains sur d'importantes surfaces : Lyon, Saint-PaulTrois-Châteaux, Fréjus, Aix-en-Provence, etc. Le corpus de Rhône-Alpes est relativement ancien, notamment en contexte rural où le nombre d'ensembles funéraires du Haut-Empire ayant fait l'objet d'une fouille ces dernières années est très limité, mais aussi dans de nombreuses villes : Roanne, Feurs et Vienne, par exemple. À Lyon, de nombreuses données ont été recueillies dans la seconde moitié du XIX ${ }^{\mathrm{e}}$ s., à l'époque où l'on s'intéressait surtout aux monuments et aux inscriptions et moins aux contextes et aux pratiques funéraires (Allmer, Dissard, 1887-1888), toutefois la moitié des données est postérieure à 1980 (Tranoy, 2007) ${ }^{3}$. Globalement, l'ancienneté d'une partie du corpus et surtout l'approche trop généraliste d'un certain nombre de sites fouillés plus récemment ont abouti à une documentation hétérogène caractérisée par des informations lacunaires et imprécises. Grâce aux travaux menés par L. Tranoy, Lyon est actuellement le site qui a le plus apporté à la connaissance de

3. On ne s'étonnera pas de la discordance entre notre corpus et celui de la Carte archéologique de la Gaule (Le Mer, Chomer, 2007), qui recèle de nombreuses surinterprétations, confusions, données primaires erronées et affabulations diverses. 
la topographie funéraire antique et plus largement à celle de l'articulation entre les morts et les divers systèmes fonctionnels emboîtés selon lesquels les villes s'organisent (Tranoy, 1995b). La relation spatiale entre les implantations rurales et les sépultures est en revanche moins documentée. Des opérations récentes ont cependant fourni des données qui montrent une grande diversité des situations, comme SaintFréjus à Marennes et Saint-Priest (Rhône), Voiron (Isère), Béligneux (Ain), Château-Gaillard (Ain), Villette-d'Anthon (Isère) et Montalieu-Vercieu (Isère), pour le Haut-Empire, ou le Pillon à Marennes (Rhône) et le Trillet à Meyzieu (Rhône) pour le Bas-Empire (Blaizot et al., 2001). Dans bien des cas, l'habitat en relation avec l'ensemble funéraire est seulement soupçonné sur la base de prospections pédestres ou de découvertes fortuites. Plusieurs séries restent non étudiées, comme celle de Villa Montel évoquée plus haut, de Chapeau-Rouge (Lyon), de Saint-Fréjus à Marennes dans le Rhône et d'autres de moindre envergure, qui proviennent de petites opérations effectuées ponctuellement en contexte périurbain. Pour beaucoup, les données demanderaient à être réanalysées et les sites réinterprétés. Sauf dans le secteur alpin, l'occupation funéraire du second âge du Fer est sousreprésentée dans la région, y compris jusqu'à la période augustéenne. À Lyon, on ne compte qu'un seul ensemble funéraire, en outre mal daté au sein de phases probablement anciennes de La Tène, les quelques mentions de mobilier pouvant se rapporter à des dépôts funéraires provenant de vieilles découvertes très peu documentées.

En Auvergne, tous les contextes périurbains et les ensembles tardo-antiques se rapportent à des découvertes anciennes (Blaizot, sous presse). Les données relatives aux ensembles périurbains de Clermont-Ferrand et aux espaces funéraires de Lezoux ont été réunies dans le cadre d'une thèse soutenue en 1982 (Mondanel, 1982) ; ce travail a le mérite d'inventorier les sites et les structures funéraires fouillés anciennement, mais les informations sont trop succinctes pour pouvoir être réintégrées dans la problématique actuelle, voire pour être réanalysées en l'état. Pour le Bas-Empire, nous ne disposons que de trois inhumations récemment fouillées sur le site de la GrandeBorne à Clermont-Ferrand. Bien que par le passé, plusieurs grands ensembles aient été découverts à la Maison Blanche sur la commune de Pardines (Desforges, Fournier, 1931), à Pérignat-lès-Sarliève (Desforges, 1956) et aux Martresd'Artière (Périchon, Chopelain, 1970), les conditions de fouille et d'enregistrement, qui ne décrivent aucun assemblage, ne permettent pas d'exploiter ces sources, d'autant plus que dans tous les cas le mobilier a été dispersé ou vendu par les inventeurs. Les données récentes relatives aux espaces funéraires ruraux du Haut-Empire proviennent du bassin de Clermont-Ferrand et concernent majoritairement le $\mathrm{II}^{\mathrm{e}} \mathrm{s}$. Elles sont actuellement en cours de réexamen et de publication ; dans ce cadre, les nombreuses données anciennes seront évaluées, notamment celles du site des Martres-de-Veyre, dont notre objectif est d'en réaliser l'étude exhaustive. Les ensembles funéraires exhumés dans l'Allier (le Plansionnier à Saint-Prix, Lapalisse) n'ont pas fait l'objet d'une approche adaptée, tandis que le Cantal et la Haute-Loire, régions peu touchées par l'archéologie préventive, ne livrent que des informations anciennes, disparates et imprécises. On note, comme en Rhône-Alpes, une indigence de données relatives au I ${ }^{\mathrm{er}} \mathrm{s}$. av. J.-C. qui s'étend ici jusqu'à la moitié du $\mathrm{I}^{\mathrm{er}} \mathrm{s}$. de notre ère, alors que les $\mathrm{II}^{\mathrm{e}} \mathrm{s}$. av. et apr. J.-C. sont très bien représentés.

En Provence, ce sont les ensembles funéraires périurbains qui sont à ce jour les mieux documentés grâce aux fouilles extensives réalisées dans les années 1980 et 1990 à Fréjus (Pauvadou : 176 tombes ; Saint-Lambert : 244 tombes), à Marseille (Sainte-Barbe : 436 tombes ; Moliner et al., 2003) ou à Aix-en-Provence (ZAC Sextius-Mirabeau : environ 60 tombes, $c f$. Nin et al., 2006) et, plus récemment, à Orange (site des Fourches-Vieilles dont les tombes sont en cours d'étude). La situation reste néanmoins contrastée puisque, pour les autres villes de la région, on ne dispose que d'une documentation ancienne ou de données récentes très ponctuelles, comme par exemple à Nice, Apt, Cavaillon ou Vaison-la-Romaine. Cette documentation éparse a dans quelques cas fait l'objet d'un recensement et d'une analyse globale à portée essentiellement topographique dans les volumes de la Carte archéologique de la Gaule, comme celui de Vaison (synthèse proposée par J.-C. Leyraud dans Provost, Meffre, 2003), ou dans l'Atlas topographique des villes de Gaule méridionale (par N. Nin dans Guyon et al., 1998). En milieu rural, depuis les travaux précurseurs de Georges Bérard (1961 et 1980) et de Raymond Boyer (Boyer et al., 1986) dans le Var, les ensembles ayant abouti à une publication détaillée ne sont pas nombreux. Les données disponibles proviennent principalement du Vaucluse, du Var et, pour le $\mathrm{I}^{\mathrm{er}}$ s. av. J.-C., de la basse vallée du Rhône et des Alpilles.

Sur l'autre rive du Rhône, en Languedoc-Roussillon, la plupart des données se rapportent au Languedoc oriental, le déséquilibre étant particulièrement marqué pour la période des $\mathrm{II}^{\mathrm{e}} \mathrm{s}$. et $\mathrm{I}^{\mathrm{er}} \mathrm{s}$. av. J.-C. Pour cette période, la documentation la plus récente est essentiellement nîmoise (Bel, à paraître). Contrairement à la situation provençale, les contextes funéraires périurbains sont peu et mal docu- 
mentés. La plupart des données récentes proviennent, là encore, de Nîmes, mais ne sont pas encore publiées. Les ensembles ruraux sont mieux connus mais, globalement, la documentation funéraire utilisable pour le Haut-Empire dans cette région est encore bien modeste (une centaine de tombes ou structures funéraires seulement).

En région Centre, les gisements funéraires du HautEmpire sont nombreux au regard du nombre de sites mentionnés par la Carte archéologique nationale, mais la plupart ont été fouillés au cours du XIX ${ }^{\mathrm{e}} \mathrm{s}$. (Limousin) et les informations restent succinctes. La tombe de Fontvieille fouillée en 1970 à Vareilles, dans la Creuse, a été récemment réexaminée et publiée (Dussot et al., 2001-2002). Parmi les opérations plus proches, on peut mentionner le site du Champ de l'Image à Saint-Marcel/Argentomagus (Allain et al., 1992) et ceux de Saint-Martin-des-Champs et de Lazenay à Bourges (Cher), des Varnes à Bruère-Allichamps (Cher), du Vieux Domaine à Vierzon (Cher) étudiés dans le cadre d'une thèse récente (Durand, 2005). Les sépultures de l'Indre, réétudiées dans un travail universitaire, ont fait l'objet de deux articles (Brissaud, 1990 et 1993). L'archéologie préventive a récemment exhumé au moins quatre sites dans le cadre du tracé linéaire de l'A85, dont celui déjà cité du Vieux Domaine à Vierzon (en 2001, dans le Cher), deux à Gy-en-Sologne (les Mahis et le Grand Saulé, en 2001, dans le Loir-et-Cher) et le dernier à Billy (les Cloubeaux, en 2001, dans le Loiret-Cher). Récemment, viennent d'être publiés les Béziaux à Langeais en Indre-et-Loire (Guiot, 2003), ainsi que, dans un volume collectif de la Revue archéologique du Centre de la France (Ensembles funéraires gallo-romains de la région Centre, 2006) : Tavant (Indre-et-Loire), Esvres (Indre-et-Loire), le Chemin du Plessis à Neung-sur-Beuvron (Loir-et-Cher) et les Mahis à Gy-en-Sologne. Dans le cadre du programme initié sur les agglomérations secondaires, une recherche spécifique est menée sur les ensembles funéraires. Citons encore trois articles récents relatifs à des secteurs un peu en marge de la zone étudiée, l'un sur une sépulture fouillée à Chauxde-Coudert sur la commune de Saint-Martial-de-Gimel, en Corrèze (Lintz, Soulier, 1993), le deuxième aux Champs Rougis à Muron en Charente-Maritime (Bolle et al., 1995) et le troisième à la Betoulle, commune de Saint-Maurice-laSouterraine, dans la Creuse (Roger, 1995).

La plupart des mentions funéraires du Haut-Empire dans la partie orientale de la région Midi-Pyrénées sont très anciennes et les descriptions sont difficiles à interpréter en l'absence d'une documentation fiable et raisonnée. Le corpus présente de nombreuses incertitudes, à commencer par les mentions de «puits funéraires » ou «d'incinéra- tions » relatives à des structures pour lesquelles aucun os humain, brûlé ou non, n'est explicitement mentionné. Les auges dites " cinéraires ", retrouvées en remploi dans les églises, faussent les inventaires, leur fonction initiale n'étant suggérée que par la présence d'un ensemble funéraire à crémation situé dans l'environnement proche de l'église dans laquelle elles ont été trouvées. La vérification des sources permet d'éliminer une grande partie des occurrences dont le nombre est finalement inférieur à dix pour l'ensemble de la région, sachant que les données véritablement exploitables réduisent encore le corpus. Il convient de préciser qu'aucun ensemble funéraire du Haut-Empire n'a fait l'objet d'une publication détaillée ; même celui de la Vayssière (L'Hospitalet-du-Larzac, Aveyron), fouillé de 1981 à 1986, qui demeure un site de référence pour la région, n’a été publié que partiellement sous la forme de quelques notices de catalogues d'expositions ou dans le cadre d'une table ronde et d'un colloque (Vernhet, 1987a, 1987 b et 1991). En l'absence de fouilles programmées sur les ensembles funéraires de l'Antiquité - signalons toutefois les travaux menés sur les piles funéraires à l'initiative du Service régional de l'archéologie -, c'est à l'archéologie préventive que l'on doit l'essentiel des connaissances sur les pratiques funéraires du Haut-Empire, que ce soit en contexte urbain (voir le site des allées François-Verdier à Toulouse par exemple) ou rural (voir les travaux liés au tracé autoroutier A20 : le site de Larsou à Réalville dans le Tarn-et-Garonne ; le site du Grand Noble 3 à Blagnac en Haute-Garonne). Un inventaire et un bilan critique de la documentation sont actuellement en cours par P. Georges et C. Amiel de l'Inrap.

En conclusion, le corpus sur lequel s'appuie ce dossier est assez inégal, tant dans la qualité des données exploitables, que par les contextes et la chronologie représentés. Ces facteurs rendent irréalisables l'analyse des faits à l'échelle des cités et limitent considérablement l'approche des identités régionales et les possibilités de comparaisons extrarégionales ; ces questions ne sont donc évoquées qu'occasionnellement et un grand nombre de données n'ont pu s'inscrire dans ce dossier que pour illustrer une question ponctuelle.

Au-delà du réexamen des données primaires et de la présentation précise des configurations archéologiques rencontrées, ce dossier aborde différentes orientations. Précisons qu'il ne s'agit pas ici d'imposer un modèle unique de lecture des vestiges funéraires, mais de livrer un état de notre réflexion, fruit d'une collaboration de diverses compétences mises au service de configurations régionales. 
Il n'est pas question non plus de militer pour des études exhaustives, puisque rares sont les sites dont l'état de conservation ou la surface d'investigation nécessitent d'en étudier tous les aspects avec précision. Chaque structure, chaque site porte en soi un potentiel très spécifique et les objectifs poursuivis ici sont de présenter l'étendue de la problématique funéraire et de proposer des pistes d'orientation, de manière à ce que les stratégies et les études puissent être mises en œuvre en fonction de ce que chaque site possède de caractéristique.
On ne peut achever cette introduction sans rappeler que la manière dont nous avons choisi d'aborder la question nous a demandé de réanalyser, réagencer et même réinterpréter la majeure partie des données, les nôtres comprises, tout cela dans un emploi du temps le plus souvent imprévisible et parfois bien lourd. C'est pourquoi, avant d'ouvrir ce dossier, nous saluons l'infinie patience de Paul Van Ossel et la bienveillance du comité de rédaction de la revue, qui ont dû jongler bien souvent avec notre calendrier vacillant et chaotique. 